\title{
Vacuum Energy Fluctuations, The Induced Cosmological Constant and Cosmological Reconstruction in Non-Minimal Modified Gravity Models
}

\author{
Guido Cognola $^{\mathrm{a},{ }^{*}, \text { Emilio Elizalde }^{\mathrm{b}} \text { Shin'ichi Nojiri }}{ }^{\mathrm{c}}$ and Sergei D. Odintsov ${ }^{\mathrm{b}, \mathrm{d}}$ \\ ${ }^{a}$ Dipartimento di Fisica, Università di Trento, and Istituto Nazionale di Fisica Nucleare, Gruppo Collegato di Trento, \\ Italia \\ ${ }^{b}$ Consejo Superior de Investigaciones Cientficas (ICE/CSIC) and Institut d'Estudis Espacials de Catalunya (IEEC), \\ Campus UAB, Facultat Ciències, Torre C5-Par-2a pl, E-08193 Bellaterra (Barcelona) Spain \\ ${ }^{c}$ Department of Physics, Nagoya University, Nagoya 464-8602, Japan \\ ${ }^{d}$ ICREA, Barcelona, Spain and Institut de Ciencies de l'Espai (IEEC-CSIC), Campus UAB, Facultat Ciències, Torre C5- \\ Par-2a pl, E-08193 Bellaterra (Barcelona) Spain
}

\begin{abstract}
The one-loop effective action for non-minimal scalar modified gravity on de Sitter background with a constant scalar field is found. The corresponding induced cosmological constant is evaluated. It is shown that quantum effects in non-minimal modified gravity may induce an early-time de Sitter universe even in the situation when such solution does not occur on the classical level. Classical reconstruction of the theory is presented in such a way that the resulting theory has a cosmological solution unifying early-time inflation with late-time acceleration.
\end{abstract}

Keywords: Dark energy, modified gravity, induced cosmological constant.

\section{INTRODUCTION}

It has become clear recently that modified gravity may suggest a very natural gravitational alternative for the unified description of the early-time and the late-time accelerating epochs of our universe (for a review and comparison of different modified gravities, see [1]). In this scenario, the universe evolution (decrease of the universe curvature) defines the gravitational sector which describes the evolution in its own turn. Starting from the first modified gravity which unifies early-time inflation with late-time acceleration, and which is consistent with the local tests [2], a number of realistic unified alternative gravities of this kind has been proposed (for a list and detailed classification, see [3]). A very interesting sub-class of modified gravities is the socalled non-minimal gravity (for a review, see [4]). That is the theory where the gravity function couples with the whole matter sector (in the Lagrangian description). It has been proven that such non-minimal models can easily describe the dark energy epoch [5].

In the present paper we consider some cosmological and related quantum aspects of a scalar theory with a nonminimal interaction with gravity. More precisely, we assume the "coupling constant" $f(R)$ between the scalar sector and gravity to depend explicitly on the scalar curvature. The oneloop effective action and the corresponding induced cosmological constant for some versions of such nonminimal gravity on the de Sitter background with a constant scalar will be found. It is demonstrated in the following that, even when starting from flat space, the quantum effects

*Address correspondence to this author at the Dipartimento di Fisica, Università di Trento, and Istituto Nazionale di Fisica Nucleare, Gruppo Collegato di Trento, Italia; Tel: +39 0461 281549; Fax: +390461 281696; E-mail: cognola@science.unitn.it induce an early-time de Sitter phase (early-time inflation) in the non-minimal gravity theory. This may be the origin of the early-time inflation in such models. The classical cosmological reconstruction for non-minimal theories is developed. Within such scheme, the possibility of the unification of the inflation with the dark energy epoch will be demonstrated.

The paper is organized as follows. In Sect. 2 we investigate the scalar non-minimal models from the classical point of view and derive the conditions for the existence of (anti-)de Sitter and Minkowski solutions. The third section is devoted to the development of the classical reconstruction scheme for the above non-minimal theory. Using such technique, the non-minimal theory unifying early-time inflation with late-time acceleration is reconstructed. The reconstruction of the models having the de Sitter solutions with a time-dependent background scalar is also done. In Sect. 4, for the scalar non-minimal model, we compute the one-loop effective action on a maximally symmetric background with constant scalar and derive the explicit "on-" and "off-shell" expressions. The one-loop, gauge-fixing independent effective action is also evaluated.

Section 5 is devoted to the calculation of the induced cosmological constant, which can be interpreted as the vacuum energy due to quantum fluctuations of the gravitational field and of the matter ones on the background manifold. The one-loop effective action of the previous section is used in this calculation. In section six we study the induced cosmological constant for several non-minimal models on the de Sitter background in the large-curvature limit. It is explicitly shown there that the one-loop effective action may induce the de Sitter space at the quantum level, even in the situation when such classical solution is absent. Some summary is presented in the final section. 


\section{SCALAR NON-MINIMAL MODIFIED GRAVITY}

We start from the model introduced in Ref. [5,6], which is characterized by a non-minimal coupling between gravity and the scalar field sector. It has been proposed as a viable model of modified gravity [5], which can describe the latetime acceleration epoch. Different aspects of such theory have been studied, including the induced cosmological constant [7], some cosmological aspects, and the appearance of an extra force [8,9], (for a review, see [4]). The action is taken to be

$$
S=-\int d^{4} x \sqrt{-g}\left[-R+f(R) L_{S}\right]
$$

where

$$
L_{S}=-\frac{1}{2} g^{i j} \partial_{i} \phi \partial_{j} \phi-V(\phi)
$$

is the Lagrangian density of a scalar field $\phi$ with an arbitrary potential $V(\phi)$. The classical field equations following from (1) read

$$
\begin{gathered}
R_{i j}-\frac{1}{2} R g_{i j}=\frac{1}{2} f(R) T_{i j}^{S}+\left[g_{i j} \mathrm{~W}-\nabla_{i} \nabla_{j}+R_{i j}\right)\left[f^{\prime}(R) L_{S}\right], \\
-\frac{1}{\sqrt{-g}} \partial_{i}\left[\sqrt{-g} f(R) g^{i j} \partial_{j} \phi\right]+f(R) V^{\prime}(\phi)=0,
\end{gathered}
$$

where

$$
T_{i j}^{S}=g_{i j}\left[\frac{1}{2} g^{r s} \partial_{r} \phi \partial_{s} \phi+V(\phi)\right]-\partial_{i} \phi \partial_{i} \phi
$$

is the standard energy-momentum tensor of the scalar field.

The action (1) has a (anti)de Sitter solution $\left(R_{0}, \phi_{0}\right)$, with constant curvature $R_{0}$ and constant field $\phi_{0}$, if the following conditions are fulfilled:

$$
R_{0}-R_{0} f^{\prime}\left(R_{0}\right) V\left(\phi_{0}\right)+2 f\left(R_{0}\right) V\left(\phi_{0}\right)=0, \quad f\left(R_{0}\right) V^{\prime}\left(\phi_{0}\right)=0 .
$$

In order to satisfy the last condition we can choose $V^{\prime}\left(\phi_{0}\right)=0$ or $f\left(R_{0}\right)=0$. We also note that, if $V\left(\phi_{0}\right)=0$, then the solution is flat, but one can have Minkowski space solution without restrictions on the potential, if $f(0)=0$.

The stability of the solutions above with respect to homogeneous perturbations can be obtained by using method of Refs. [10, 11], One easily gets

$$
\frac{1+f^{\prime}\left(R_{0}\right) V\left(\phi_{0}\right)}{R_{0} f^{\prime \prime}\left(R_{0} V\left(\phi_{0}\right)\right.}>1
$$

The field equation for the scalar field can be written in the form

$$
\mathrm{W} \phi-V^{\prime}(\phi)=0,
$$

It is interesting to note that in the case of vanishing potential there are no solutions with constant $R=R_{0}$, since in such a case from Eqs. (3) and (4) we get $\mathrm{W} \phi=0, \quad R+\left[2 f(R)-R f^{\prime}(R)\right] \frac{g^{i j} \partial_{i} \phi \partial_{j} \phi}{2}=0$.

In the FRW background, the first equation has the following form:

$$
\mathrm{W} \phi=\frac{1}{a^{3}} \frac{d}{d t}\left(a^{3} \frac{d \phi}{d t}\right)=0,
$$

which can be solved as

$$
\frac{d \phi}{d t}=C a^{-3}
$$

Here $C$ is a constant of integration and $a$ is a scale factor. Then the second equation in (9) has the following form:

$$
-\frac{2 R_{0}}{2 f\left(R_{0}\right)-R_{0} f^{\prime}\left(R_{0}\right)}=g^{i j} \partial_{i} \phi \partial_{j} \phi=-C^{2} a^{6} .
$$

In the de Sitter background, the 1.h.s. is a constant but the scale factor $a$ is not a constant in the expanding universe. Then, unless $C \neq 0$, there is not consistent solution but $C \neq 0$ implies $R=0$, which is the flat space-time.

The stability of the solution can be obtained by using [11]. We have

$$
\frac{1+a^{2} f^{\prime}\left(R_{0}\right)}{a^{2} R_{0} f^{\prime \prime}\left(R_{0}\right)}-1>0,
$$

which agrees with the standard result when $a^{2}=1$.

\section{COSMOLOGICAL RECONSTRUCTION AND THE UNIFICATION OF THE INFLATION EPOCH WITH DARK ENERGY}

In this section we consider how one can construct a classical model which reproduces an almost arbitrary time evolution of the universe. Note that a general scheme of cosmological reconstruction of modified gravity has been developed for a number of modified gravities (for review, see [12] based on the technique developed in Refs. [13, 14]). The same method will be applied below. The starting action is (1) with (2). We redefine the scalar field by using an adequate function $h$ as $\phi=h(\varphi)$. Then Eq. (2) has the following form:

$$
\begin{aligned}
& L_{S}=-\frac{1}{2} \omega(\varphi) g^{i j} \partial_{i} \varphi \partial_{j} \varphi-\tilde{V}(\varphi), \quad \omega(\varphi) \equiv h^{\prime}(\varphi)^{2}, \\
& \tilde{V}(\varphi) \equiv V(h(\varphi)) .
\end{aligned}
$$

Especially by choosing $h$ properly, one may identify $\varphi$ as a time coordinate.

In the FRW background with a flat spatial part, the equation corresponding to the first FRW equation has the following form:

$$
\begin{aligned}
& 3 H^{2}=\frac{f(R)}{2}\left(\frac{\omega(\varphi)}{2} \dot{\varphi}^{2}+\tilde{V}(\varphi)\right)-3\left\{H \frac{d}{d t}-\left(\dot{H}+H^{2}\right)\right\} \\
& \left\{f^{\prime}(R)\left(\frac{\omega(\varphi)}{2} \dot{\varphi}^{2}-\tilde{V}(\varphi)\right)\right\} .
\end{aligned}
$$

Here the scalar curvature is $R=6 \dot{H}+12 H^{2}$. On the other hand, by variation over $\varphi$, we find

$$
0=f(R)\left(\frac{\omega^{\prime}(\varphi)}{2} \dot{\varphi}^{2}-\tilde{V}^{\prime}(\varphi)\right)-\left(\frac{d}{d t}+3 H\right)\{f(R) \omega(\varphi) \dot{\varphi}\} .
$$


Identifying $\varphi$ as a cosmological time coordinate $\varphi=t$, Eqs. (15) and (16) can be rewritten as

$$
\begin{gathered}
3 H^{2}=\frac{f(R(t))}{2} \rho(t)-3\left\{\frac{d}{d t}-\left(\dot{H}+H^{2}\right)\right\}\left(f^{\prime}(R(t)) p(t)\right), \\
0=f(R(t)) p^{\prime}(t)-\left(\frac{d}{d t}+3 H\right)\{f(R(t))(p(t)+\rho(t))\} .
\end{gathered}
$$

Here

$$
\rho(\varphi) \equiv \frac{\omega(\varphi)}{2}+\tilde{V}(\varphi), \quad p(\varphi) \equiv \frac{\omega(\varphi)}{2}-\tilde{V}(\varphi) .
$$

In the following, we consider the cosmological reconstruction of the model (for a general review of reconstruction in different modified gravities, see [12]), that is, given the specific time-development of the scale factor $a$ or the Hubble rate $H$, we construct a model which reproduces such evolution. Although only one function $a=a(t)$ or $H=H(t)$ is given, we have two functions $f(R)$ and $V(\phi)$ in the action (1). Then, in principle one of the functions can be arbitrary. For example, if we choose $f(R)=1$, we have the usual scalar-tensor theory [15]. Now we choose

$$
f(R) a^{3}=f_{0}
$$

Here $f_{0}$ is a constant. If one solves $R=R(t)$ with respect to $t$ as $t=T(R)$ for a given scale factor $a=a(t), f(R)$ is explicitly given by $f(R)=f_{0} / a(T(R))^{3}$. For example, the scale factor of the universe with one kind of perfect fluid, with constant EoS parameter $w$, is given by $a \propto t^{2 / 3(1+w)}$; then $F(R) \propto R^{-1 / 3(1+w)}$. Note that we cannot choose (20) for the de Sitter solution with $f(R)$ being a constant, since $R$ is a constant but $a$ is not in de Sitter universe. In the asymptotic de Sitter universe, where $R$ is not exactly constant, we can choose (20) as we will later see.

For given $a=a(t)$ or $H=H(t)$, which yields $R=R(t)$, Eqs. (17) and (18) can be solved with respect to $\rho(\phi)$ and $p(\phi)$, as follows

$$
\begin{gathered}
\rho(\varphi)=\left[\frac{6 a(t)^{3}}{f_{0}}\left\{H(t)^{2}-\frac{Q(t)}{a(t)^{3}}\right\}\right]_{t=\varphi}, \\
p(\varphi)=-\left[a(t) H(t) \int \frac{Q\left(t^{\prime}\right) d t^{\prime}}{a\left(t^{\prime}\right)^{4} H\left(t^{\prime}\right)^{2}}\right]_{t=\varphi}, \\
{ }^{t} Q(t) \equiv \int d t^{\prime} a\left(t^{\prime}\right)^{3}\left(2 H\left(t^{\prime}\right) \dot{H}\left(t^{\prime}\right)+3 H\left(t^{\prime}\right)^{3}\right) .
\end{gathered}
$$

From $\rho(\varphi)$ and $p(\varphi)$, one finds the explicit form of $\omega(\varphi)$ and $\tilde{V}(\varphi)$, by using (19).

As an example, we consider the following Hubble rate

$$
H(t)=\frac{H_{L} \mathrm{e}^{\alpha t}+\beta H_{I}}{\mathrm{e}^{\alpha t}+\beta} .
$$

Here, $H_{L}, H_{I}, \alpha$, and $\beta$ are positive constants. When $t \rightarrow-\infty$, we find $H(t) \rightarrow H_{I}$, which may be identified with the inflation of the early universe and, when $t \rightarrow+\infty$, $H(t) \rightarrow H_{L}$, which may be identified with the accelerated expansion of the late universe. Eq. (24) yields

$$
\begin{aligned}
& a(t)=a_{0} \mathrm{e}^{H_{I} t}\left(\mathrm{e}^{\alpha t}+\beta\right)^{\left(H_{L}-H_{I}\right) / \alpha}, \\
& R(t)=\frac{12 H_{L}^{2} \mathrm{e}^{2 \alpha t}+\left\{24 \beta H_{L} H_{I}+6 \alpha \beta\left(H_{L}-H_{I}\right)\right\} \mathrm{e}^{\alpha t}+12 \beta^{2} H_{I}^{2}}{\left(\mathrm{e}^{\alpha t}+\beta\right)^{2}},
\end{aligned}
$$

which give the forms of $f(R), \omega(\varphi)$, and $\tilde{V}(\varphi)$ by using (19), (20), (21), and (22).

e now consider the asymptotic form of $f(R), \omega(\varphi)$, and $V(\varphi)$, in the limit $t \rightarrow \pm \infty$, for the model in (24). For simplicity, we may choose $\alpha=H_{1}$ and $\beta=1$. First, we consider the behaviors of $f(R), \omega(\varphi)$, and $V(\varphi)$ when $t \rightarrow-\infty$. Since $H_{I} \gg H_{L}$, we find

$$
\begin{aligned}
& H(t): H_{I}\left(1-\mathrm{e}^{H_{I} t}\right), \quad a(t): a_{0} \mathrm{e}^{H_{I^{t}}}\left(1-\mathrm{e}^{H_{I^{t}}}\right), \\
& R: 12 H_{I}^{2}\left(1-\frac{5}{2} \mathrm{e}^{H_{I} I^{t}}\right),
\end{aligned}
$$

which give

$$
\begin{aligned}
& f(R): \frac{f_{0}}{a_{0}^{3}}\left\{\frac{2}{5}\left(1-\frac{R}{12 H_{I}^{2}}\right)\right\}^{-3}\left\{1+\frac{6}{5}\left(1-\frac{R}{12 H_{I}^{2}}\right)\right\}, \\
& \rho(\varphi): \mathrm{O}\left(\mathrm{e}^{5 H^{t}}\right), \quad p: 1-H_{I}\left(\varphi-\varphi_{0}\right) \mathrm{e}^{H_{I^{\varphi}}} .
\end{aligned}
$$

Here $\varphi_{0}$ is a constant of integration. Then, one gets

$$
\omega(\varphi):-\frac{\tilde{V}(\varphi)}{2}: 1-H_{I}\left(\varphi-\varphi_{0}\right) \mathrm{e}^{H_{I} \varphi}
$$

Note that $R$ could be large but always $R<12 H_{I}^{2}$.

On the other hand, when $t \rightarrow+\infty$ so that $\mathrm{e}^{-H_{L} t}=\frac{H_{L}}{H_{I}}=1$, we find

$$
\begin{aligned}
& H(t): H_{L}+H_{I} \mathrm{e}^{-H_{I} t}, \quad a(t): a_{0} \mathrm{e}^{H_{L} t}\left(1-\mathrm{e}^{-H_{I} t}\right), \\
& R: 12 H_{L}^{2}-6 H_{I}^{2} \mathrm{e}^{-H_{I} t},
\end{aligned}
$$

which give

$$
\begin{aligned}
& f(R): \frac{f_{0}}{a_{0}^{3}}\left(\frac{12 H_{L}^{2}-R}{6 H_{I}^{2}}\right)^{3}\left(1-\frac{R}{3 H_{I}^{2}}\right), \\
& \rho(\varphi): \mathrm{O}\left(\mathrm{e}^{\left(3 H_{L}-H_{I}\right) t}\right), \quad p: \frac{H_{L}}{H_{I}}+\mathrm{e}^{-H_{I} t} .
\end{aligned}
$$

Then, one obtains 


$$
\omega(\varphi):-\frac{\tilde{V}(\varphi)}{2}: \frac{H_{L}}{H_{I}}+\mathrm{e}^{-H_{I} t} .
$$

Thus, the function interpolating between the two functions $f$ above describes inflation, when $t \rightarrow-\infty$, and the late-time acceleration epoch, when $t \rightarrow+\infty$. Its explicit form is however quite complicated. But in any case, such theory describes a perfectly unified evolution from the earlytime inflation era to the late-time acceleration one.

Instead, when we choose the condition (20), if we consider a general $f(R)$, by deleting $\rho(t)$ in (17) and (18), we obtain

$$
\begin{aligned}
& 0=p(t)\left(-3 H f(R(t))-\frac{d f(R(t))}{d t}\right)- \\
& \frac{6}{a(t)^{3}} \frac{d}{d t}\left(a^{4} H^{2} \frac{d}{d t}\left(\frac{f^{\prime}(R(t)) p(t)}{a(t) H(t)}\right)\right)-\left(12 H \dot{H}+18 H^{3}\right) .
\end{aligned}
$$

If $f(R)$ is properly given, Eq. (32) can be regarded as a differential equation for $p(t)$ (compare with [12, 13]. If we find the form of $p(\varphi)$, by solving (32), we also find the form of $\rho(\varphi)$ by using (19), as

$$
\rho(\varphi)=\left.\frac{6}{f(R(t))}\left[H(t)^{2}+\left(H(t) \frac{d}{d t}-\dot{H}(t)-H(t)^{2}\right)\left\{f^{\prime}(R(t)) p(t)\right\}\right]\right|_{t=\varphi} .
$$

For an example, we consider the de Sitter space where $H$ is a constant $H=H_{0}$. Then, Eq. (32) reduces to

$$
0=\frac{d^{2} p(t)}{d t^{2}}+2 H_{0} \frac{d p(t)}{d t}+\left(-3 H_{0}^{2}+\frac{f\left(12 H_{0}^{2}\right)}{2 f^{\prime}\left(12 H_{0}^{2}\right)}\right) p(t)+\frac{3 H_{0}^{2}}{f^{\prime}\left(12 H_{0}^{2}\right)}
$$

which can be solved as

$$
\begin{aligned}
& p(t)=C_{+} \mathrm{e}^{\lambda_{+} t^{t}}+C_{-} \mathrm{e}^{\lambda_{-} t}+\frac{3 H_{0}^{2}}{6 H_{0}^{2} f^{\prime}\left(12 H_{0}^{2}\right)+f\left(12 H_{0}^{2}\right)}, \\
& \lambda_{ \pm} \equiv H_{0} \pm \sqrt{4 H_{0}^{2}-\frac{f\left(12 H_{0}^{2}\right)}{2 f^{\prime}\left(12 H_{0}^{2}\right)}} .
\end{aligned}
$$

Here, the $C_{ \pm}$are constants of integration. Then, Eq. (33) gives

$$
\begin{aligned}
& \rho(t)=\frac{6 H_{0}^{2}}{f\left(12 H_{0}^{2}\right)}\left(\frac{3 H_{0}^{2} f^{\prime}\left(12 H_{0}^{2}\right)+f\left(12 H_{0}^{2}\right)}{6 H_{0}^{2} f^{\prime}\left(12 H_{0}^{2}\right)+f\left(12 H_{0}^{2}\right)}+\right. \\
& \left.\left(H_{0} \lambda_{+}-H_{0}^{2}\right) C_{+} \mathrm{e}^{\lambda_{+} t^{t}}+\left(H_{0} \lambda_{-}-H_{0}^{2}\right) C_{+} \mathrm{e}^{\lambda_{-} t}\right) .
\end{aligned}
$$

In particular, in the case when the constants of integration vanish, $C_{\omega}=0$, both $p(t)$ and $\rho$ become constant, and then $\omega$ and $V$ are given by

$$
\begin{aligned}
& \omega=\rho+p=\frac{9 H_{0}^{2}\left(2 H_{0}^{2} f^{\prime}\left(12 H_{0}^{2}\right)+f\left(12 H_{0}^{2}\right)\right)}{f\left(12 H_{0}^{2}\right)\left(6 H_{0}^{2} f^{\prime}\left(12 H_{0}^{2}\right)+f\left(12 H_{0}^{2}\right)\right)}, \\
& V=\frac{\rho-p}{2}=\frac{3 H_{0}^{2}\left(6 H_{0}^{2} f^{\prime}\left(12 H_{0}^{2}\right)+f\left(12 H_{0}^{2}\right)\right)}{f\left(12 H_{0}^{2}\right)\left(6 H_{0}^{2} f^{\prime}\left(12 H_{0}^{2}\right)+f\left(12 H_{0}^{2}\right)\right)} .
\end{aligned}
$$

We should not fix $\varphi$, and therefore $\phi$ is not a constant

$$
\varphi=t, \quad \phi=\sqrt{\omega} t+\phi_{0} .
$$

Then the above de Sitter solution does not correspond to any of the four classes of dS solutions (42) for which the one-loop effective action is evaluated in the next section.

\section{ONE-LOOP QUANTIZATION AROUND A MAXIMALLY SYMMETRIC SPACE}

We will here perform the one-loop quantization of the (Euclidean) classical scalar model we have discussed above. One-loop contributions are certainly important, especially during the inflationary phase, but as it has been shown in [16], they also provide a very powerful method in order to study the stability of the solutions.

In accordance with the background field method, we consider small fluctuations $\left(h_{i j}, \varphi\right)$ of the fields $\left(g_{i j}, \phi\right)$, around a maximally symmetric space. Then,

$$
g_{i j} \rightarrow g_{i j}+h_{i j}, \quad \phi \rightarrow \phi+\varphi, \quad R_{i j r s} \rightarrow R_{i j r s}+O\left(\nabla h_{i j}\right) .
$$

Here and in what follows $g_{i j}$ is the metric of $S O(4)$ and $R_{i j r s}$ the corresponding Riemann tensor. Thus

$$
R_{i j r s}=\frac{\Lambda}{3}\left(g_{i r} g_{j s}-g_{i s} g_{j r}\right), \quad R_{i j}=\Lambda g_{i j}, \quad R=4 \Lambda=\text { const } .
$$

As already said above, if the functions $f(R)$ and $V(\phi)$ satisfy the conditions $(6)$, then $\left(g_{i j}, \phi\right)$ is a classical solution of (1) with constant $\phi=\phi_{0}$ and $R=R_{0}$. We can distinguish the following four cases:
a) $f\left(R_{0}\right)=0, \quad f^{\prime}\left(R_{0}\right) V\left(\phi_{0}\right)=1$,
$R_{0} \neq 0$,
b) $V^{\prime}\left(\phi_{0}\right)=0$
$R_{0}-R_{0} f^{\prime}\left(R_{0}\right) V\left(\phi_{0}\right)+2 f\left(R_{0}\right) V\left(\phi_{0}\right)=0$,
$R_{0} \neq 0$,
c) $f(0)=0$,
$R_{0}=0$
d) $V^{\prime}\left(\phi_{0}\right)=0, \quad V\left(\phi_{0}\right)=0$,
$R_{0}=0$.

The last two cases correspond to Minkowski solutions.

Now, we perform a Taylor expansion of the action around the $S O(4)$ background manifold, up to second order in the small variables $\left(h_{i j}, \varphi\right)$. A straightforward calculation [17-19] for zero and second order contributions in the fields $\left(h_{i j}, \varphi\right)$ and disregarding total derivatives yields, respectively,

$$
L_{0}=-R-f V \Rightarrow I=\int_{S O(4)} d x^{4} \sqrt{g} L_{0}=-\frac{24 \pi^{2}[R+V f(R)]}{\Lambda^{2}}
$$

and 


$$
\begin{aligned}
& L_{2}=-\frac{1}{2} f \varphi\left(-\Delta+V^{\prime \prime}\right) \varphi-V^{\prime} \varphi\left[\frac{3 f^{\prime}}{4}\left(-\Delta-\frac{R}{3}\right)+\frac{1}{2} f\right] h+ \\
& \frac{3 f^{\prime}}{4} V^{\prime} \varphi\left(-\Delta-\frac{R}{3}\right) \Delta \sigma \\
& -\frac{3}{32} h\left[3 V f^{\prime \prime}\left(-\Delta-\frac{R}{3}\right)^{2}+V f^{\prime}\left(-\Delta-\frac{2 R}{3}\right)+\left(-\Delta+\frac{2 V f}{3}\right)\right] h \\
& +\frac{3 f^{\prime} V^{\prime}}{16} h\left(-\Delta-\frac{R}{3}\right)\left[3 f^{\prime \prime} V\left(-\Delta-\frac{R}{3}\right)+f^{\prime} V+1\right) \Delta \sigma \\
& -\frac{3}{32} \sigma\left(-\Delta-\frac{R}{3}\right)\left[3 f^{\prime \prime} V\left(-\Delta-\frac{R}{3}\right) \Delta-f^{\prime} V(-\Delta+R)+\Delta+R+2 f V\right] \Delta \sigma \\
& +\frac{R+2 f V-f^{\prime} R V}{4} \tilde{\xi}^{k}\left(-\Delta-\frac{R}{4}\right) \tilde{\xi}_{k} \\
& +\frac{1}{4} \tilde{h}^{i j}\left[-\Delta+\frac{2 R}{3}+f V+f^{\prime} V\left(-\Delta-\frac{R}{3}\right)\right] \tilde{h}_{i j}
\end{aligned}
$$

$I$ being the classical action on $S O(4)$. In contrast with Sect. (2), here $\nabla_{k}$ and $\Delta=g^{i j} \nabla_{i} \nabla_{j}$ represent the covariant derivative and the D'Alembertian in the unperturbed metric $g_{i j}$, and all functions are evaluated on the background $\left(g_{i j}, \phi\right)$. This means that $f=f(R), f^{\prime}=f^{\prime}(R)$, and so on.

For technical reasons we have also carried out the standard expansion of the tensor field $h_{i j}$ in irreducible components [20], that is

$$
h_{i j}=\tilde{h}_{i j}+\nabla_{i} \tilde{\xi}_{j}+\nabla_{j} \tilde{\xi}_{i}+\nabla_{i} \nabla_{j} \sigma+\frac{1}{4} g_{i j}(h-\Delta \sigma),
$$

where $\sigma$ is the scalar component, while $\tilde{\xi}_{i}$ and $\tilde{h}_{i j}$ are the vector and tensor components, respectively. They have the properties

$$
\nabla_{i} \tilde{\xi}^{i}=0, \quad \nabla_{i} \tilde{h}_{j}^{i}=0, \quad \tilde{h}_{i}^{i}=0 .
$$

Invariance under diffeomorphisms renders the operator in the $(h, \sigma)$ sector non-invertible. One needs to involve a gauge fixing term and a corresponding ghost compensating term. We will consider the class of gauge conditions:

$$
\chi_{k}=\nabla_{j} h_{j k}-\frac{1+\rho}{4} \nabla_{k} h,
$$

parameterized by the real parameter $\rho$ and, for gauge fixing, we choose the quite general form [21]

$$
L_{g f}=\frac{1}{2} \chi^{i} G_{i j} \chi^{j}, \quad G_{i j}=\gamma g_{i j}+\beta g_{i j} \Delta,
$$

where the term proportional to $\gamma$ is the one normally used in Einstein's gravity.

Finally, one also has to add the ghost Lagrangian [21]

$$
L_{g h}=B^{i} G_{i k} \frac{\delta \chi^{k}}{\delta \varepsilon^{j}} C^{j},
$$

where $C_{k}$ and $B_{k}$ are the ghost and anti-ghost vector fields, respectively, while $\delta \chi^{k}$ is the variation of the gauge condition due to an infinitesimal gauge transformation of the field (details can be found in Refs. [17, 21]).

In order to compute the one-loop contributions to the effective action, one has to consider the path integral for the bilinear part $L=L_{2}+L_{g f}+L_{g h}$ of the total Lagrangian and take into account the Jacobian due to the change of variables with respect to the original ones. In this way, we get [20, 21].

$$
\begin{aligned}
& Z^{(1)}=\left(\operatorname{det} G_{i j}\right)^{-1 / 2} \int D\left[h_{i j}\right] D\left[C_{k}\right] D\left[B^{k}\right] \exp \left(\int d^{4} x \sqrt{g} L\right) \\
& =\left(\operatorname{det} G_{i j}\right)^{-1 / 2} \operatorname{det} J_{1}^{-1} \operatorname{det} J_{2}^{1 / 2} \\
& \times \int D[h] D\left[\tilde{h}_{i j}\right] D\left[\tilde{\xi}^{j}\right] D[\sigma] D\left[\tilde{C}_{k}\right] D\left[\tilde{B}^{k}\right] D[c] D[b] \exp \left(\int d^{4} x \sqrt{g} L\right),
\end{aligned}
$$

where $J_{1}$ and $J_{2}$ are the Jacobians coming from the change of variables in the ghost and tensor sectors, respectively. They read [21].

$$
J_{1}=\Delta_{0}, \quad J_{2}=\left(-\Delta_{1}-\frac{R}{4}\right)\left(-\Delta_{0}-\frac{R}{3}\right) \Delta_{0},
$$

while the determinant of the operator $G_{i j}$ acting on vectors in our gauge assumes the form (up to a constant)

$$
\operatorname{det} G_{i j}=\operatorname{det}\left(\Delta_{1}+\frac{\gamma}{\beta}\right) \operatorname{det}\left(\Delta_{0}+\frac{R}{4}+\frac{\gamma}{\beta}\right),
$$

and it is trivial in the simplest case, $\beta=0$. By $\Delta_{n}$ we indicate the Laplacians acting on transverse tensors fields of order $n$.

A straightforward computation, disregarding zero modes of gravity and the multiplicative anomaly [22], leads to the one-loop contribution $Z^{(1)}(\gamma, \beta, \rho)$ to the "partition function". The result is a very complicated expression depending on the gauge parameters. As is well known, on shell---that is, when one of the conditions in (42) is satisfied-- $Z^{(1)}$ does not depend on the gauge. For the four possible cases we get, respectively,

a) $Z_{\text {on-shell }}^{(1)}=\left[\frac{\operatorname{det}\left(-\Delta_{1}-\Lambda_{0}\right)}{\operatorname{det}\left(-\Delta_{2}+\frac{2}{3} \Lambda_{0}\right)}\right]^{1 / 2}\left[\operatorname{det}\left(-\Delta_{0}-\frac{4}{3} \Lambda_{0}\right)\right]^{-1 / 2}$,
b) $Z_{\text {on-shell }}^{(1)}=\left[\frac{\operatorname{det}\left(-\Delta_{1}-\Lambda_{0}\right)}{\operatorname{det}\left(-\Delta_{2}+\frac{2}{3} \Lambda_{0}\right)}\right]^{1 / 2}\left[\operatorname{det}\left(-\Delta_{0}+V_{0}^{\prime}\right) \operatorname{det}\left(-\Delta_{0}+M_{0}^{2}\right)\right]^{-1 / 2}$,
c) $Z_{\text {on-shell }}^{(1)}=\left[\frac{\operatorname{det}\left(-\Delta_{1}\right)}{\operatorname{det}\left(-\Delta_{2}\right)}\right]^{1 / 2}$,
d) $Z_{\text {on-shell }}^{(1)}=\left[\frac{\operatorname{det}\left(-\Delta_{1}\right)}{\operatorname{det}\left(-\Delta_{2}\right)}\right]^{1 / 2}\left[\operatorname{det}\left(-\Delta_{0}+V_{0}^{\prime}\right)\right]^{-1 / 2}$.

Here we have introduced an effective mass

$$
M_{0}^{2}=\frac{8 f_{0}^{\prime \prime} \Lambda_{0}^{2} V_{0}-f_{0} V_{0}-4 \Lambda_{0}}{6 f_{0}^{\prime \prime} \Lambda_{0} V_{0}},
$$


$\Lambda=\Lambda_{0}=R_{0} / 4, R_{0}$ being the solution of (42), and $f_{0}=f\left(R_{0}\right), V_{0}=V\left(\phi_{0}\right)$, and so on. We see that, in the second case $(b)$, the scalar potential explicitly appears in the expression of the on-shell one-loop effective action. It looks like, in the first case $(a)$, the scalar field does not give a contribution, but this is not so, since in pure Einsteinian gravity with a cosmological constant the last term in equation $(a)$ is not present. This is a contribution due to the non-minimal coupling between gravity and matter.

From expressions in (52) one can obtain the stability condition of the corresponding de Sitter solution, by requiring that all Laplacian-like operators involved have no negative eigenvalues [16]. For example, the stability of de Sitter solution corresponding to the second case $(b)$ is assured if $V_{0}^{\prime \prime}>0$ and $M_{0}^{2}>0$, which are the conditions one obtains by the classical expression (13). On the contrary, the solution corresponding to the first case $(a)$ is always unstable, in contrast with what one obtains from (13). But it has to be stressed that the classical condition (13) has been derived by considering only homogeneous perturbations.

As already explained above, the off-shell one-loop contribution to the partition function, in the most general case, is a very complicated expression we shall not write here explicitly. For the sake of simplicity we will restrict the gauge parameters by putting $\beta=0$ and we shall consider only the two special cases $V(\phi)=V^{\prime}(\phi)=0, V^{\prime \prime}(\phi) \neq 0$ and, alternatively, $f(R)=0$, but $f^{\prime}(R) \neq 0, f^{\prime \prime}(R) \neq 0$. In this way, the expression of $Z^{(1)}(\gamma, 0, \rho)$ notably simplifies. We get

$$
\begin{aligned}
& Z^{(1)}(\gamma, 0, \rho)=\operatorname{det}\left(-\Delta_{0}-\frac{4 \Lambda}{3-\rho}\right) \operatorname{det}\left(-\Delta_{1}-\Lambda\right) \\
& \times\left[\operatorname{det}\left(-\Delta_{2}+\frac{8}{3} \Lambda\right) \operatorname{det}\left(-\Delta_{1}-\Lambda\left[1-\frac{2}{\gamma}\right]\right)\right. \\
& \times\left[\operatorname{det}\left(-\Delta_{0}-\frac{4 \Lambda\left[2(\gamma-1)+\gamma(1-\rho)^{2}\right]}{\gamma(3-\rho)^{2}}\right) \operatorname{det}\left(-\Delta_{0}+V^{\prime \prime}\right)\right]^{-1 / 2}, \\
& Z^{(1)}(\gamma, 0, \rho)=\operatorname{det}\left(-\Delta_{0}-\frac{4 \Lambda}{3-\rho}\right) \operatorname{det}\left(-\Delta_{1}-\Lambda\right) \\
& \times\left[\operatorname{det}\left(-\Delta_{2}-\frac{4 \Lambda\left(f^{\prime} V-2\right)}{3\left(f^{\prime} V+1\right)}\right) \operatorname{det}\left(-\Delta_{1}-\Lambda+\frac{2 \Lambda\left(f^{\prime} V-1\right)}{\gamma}\right)\right]^{-1 / 2} \\
& \times\left[\operatorname{det}\left(-\Delta_{0}-\frac{4 \Lambda}{3}\right) \operatorname{det}\left(-\Delta_{0}-\frac{\Lambda}{3} q_{1}\right) \operatorname{det}\left(-\Delta_{0}-\frac{\Lambda}{3} q_{2}\right)\right]^{-1 / 2} .
\end{aligned}
$$

Here we have set $R=4 \Lambda$, since we are interested in the evaluation of the induced cosmological constant. The quantities $q_{1}, q_{2}$ are the roots of the second-order algebraic equation $q^{2}+c_{1} q+c_{0}=0$, where $c_{0}=\frac{144\left(f^{\prime} V-1+\gamma\right)}{\gamma(3-\rho)^{2}}, \quad c_{1}=-\frac{24\left[f^{\prime} V-1+\gamma(3-\rho)\right]}{\gamma(3-\rho)^{2}}$.

It is well known that the one-loop effective action in gravity, as well as in non-abelian gauge theories, is a gauge dependent one. One can use the gauge-fixing independent effective action (for a review, see [21]) in order to solve gauge dependence problem. It is known that such gaugefixing independent action agrees with the standard one computed in the Landau gauge $(\gamma=\infty, \beta=0, \rho=1)$ (but only in the one-loop approximation). Then, we write down the expressions (54) and (55) also in such a particular important gauge. We get, respectively,

$$
\begin{aligned}
& Z^{(1)}(\infty, 0,1)=\left[\frac{\operatorname{det}\left(-\Delta_{1}-\Lambda\right)}{\operatorname{det}\left(-\Delta_{2}+\frac{8}{3} \Lambda\right)} \frac{\operatorname{det}\left(-\Delta_{0}-2 \Lambda\right)}{\operatorname{det}\left(-\Delta_{0}+V^{\prime \prime}\right)}\right]^{1 / 2}, \\
& V(\phi)=0=V^{\prime}(\phi)=0,
\end{aligned}
$$

$$
Z^{(1)}(\infty, 0,1)=\left[\frac{\operatorname{det}\left(-\Delta_{1}-\Lambda\right)}{\operatorname{det}\left(-\Delta_{2}-\frac{4 \Lambda\left(f^{\prime} V-2\right)}{3\left(f^{\prime} V+1\right)}\right)}\right]^{1 / 2}\left[\operatorname{det}\left(-\Delta_{0}-\frac{4}{3} \Lambda\right)\right]^{-1 / 2},
$$

$f(R)=0$.

Thus, we have obtained the one-loop effective action for non-minimal modified gravity on the de Sitter background with a constant scalar field.

\section{VACUUM ENERGY AND THE INDUCED COSMOLOGICAL CONSTANT}

In this section we will compute the induced cosmological constant for the last two cases, Eqs. (54) and (55), discussed in the previous section. We start with the first, Eq. (54), which represents the one-loop contribution to the partition function of a massive scalar field with background solution $\phi=0$, non-minimally coupled to gravity through the arbitrary function $f$, which depends on gravity through the scalar curvature. The only classical solution with constant curvature of this model is the Minkowskian one $\left(\Lambda_{0}=0\right)$, since we are dealing with the case (d) and, in fact, when $\Lambda=\Lambda_{0}=0$ we recover the last equation in (52). In this case, the one-loop effective action has a solution with constant curvature $R=4 \Lambda, \Lambda$ being the induced cosmological constant, which is related to the vacuum energy generated by the quantum fluctuations of the gravitational and scalar fields. In order to calculate this vacuum energy, we impose the one-loop effective action $\Gamma$ to have a solution with constant curvature $R=4 \Lambda$. Correspondingly, $\Lambda$ has to satisfy the equation

$$
\frac{\partial \Gamma}{\partial \Lambda}=\frac{\partial\left(I+\Gamma^{(1)}\right)}{\partial \Lambda}=0,
$$

where $I$ is the classical action in (43), and $\Gamma^{(1)}$ the oneloop contribution. They read

$$
I=-\frac{96 \pi^{2}}{\Lambda}, \quad \Gamma^{(1)}=-\log Z_{(\gamma, 0, \rho)}^{(1)} .
$$


Of course, Eq. (59) applied to the classical action $I$ gives rise (in our case) only to a vanishing cosmological constant $\Lambda_{0}$.

The main part of the effective action, $\Gamma^{(1)}$, can be conveniently evaluated by making use of zeta-function regularization and, specifically, by computing the zetafunctions $\zeta\left(s \mid L_{n}\right)$ related to the differential-elliptic Laplace-like operators $L_{n}[23,24]$. Using the same notations as in Ref. [17], we get

$$
\begin{aligned}
& \Gamma^{(1)}=Q_{0}\left(\frac{9}{4}+\frac{12}{3-\rho}\right)+Q_{1}\left(\frac{25}{4}\right)-\frac{1}{2} Q_{1}\left(\frac{25}{4}-\frac{6}{\gamma}\right)-\frac{1}{2} Q_{2}\left(\frac{-15}{4}\right) \\
& -\frac{1}{2} Q_{0}\left(\frac{9}{4}+\frac{12\left[2(\gamma-1)+\gamma(1-\rho)^{2}\right]}{\gamma(3-\rho)^{2}}\right)-\frac{1}{2} Q_{0}\left(\frac{9}{4}-\frac{3 m^{2}}{\Lambda}\right),
\end{aligned}
$$

where

$$
\begin{gathered}
Q_{n}(\alpha)=\zeta^{\prime}\left(0 \mid L_{n} / \mu^{2}\right), \quad L_{n}=-\Delta_{n}-\frac{\Lambda}{3}\left(\alpha-\alpha_{n}\right), \\
\zeta^{\prime}\left(0 \mid L_{n}\right)=\lim _{s \rightarrow 0} \frac{d}{d s} \zeta\left(s \mid L_{n}\right)=-\operatorname{logdet} L_{n},
\end{gathered}
$$

and $m^{2}=V^{\prime \prime}, \alpha_{0}=9 / 4, \alpha_{1}=13 / 4, \alpha_{2}=17 / 4$.

From (59), we obtain the equation for the induced cosmological constant $\Lambda$ [20]. In order to get it, we first observe that, even when the $Q_{n}$-functions do not explicitly depend on $\Lambda$, they provide contributions to the induced cosmological constant through the corresponding logarithmic term(see also Appendix in Ref. [19]), since

$$
\frac{d Q_{n}(\alpha)}{d \Lambda}=\frac{F_{\alpha}(0)+b_{0}+b_{1} \alpha+\frac{1}{2} b_{2} \alpha^{2}}{\Lambda}+Q_{n}^{\prime}(\alpha) \frac{d \alpha}{d \Lambda} .
$$

A trivial example of this kind is the one corresponding to pure Einstein gravity. For such case, $\Gamma^{(1)}$ is given by equation (61) without the last term on the right hand side. Then, none of the $Q_{n}$-functions depends on $\alpha$, and we get

$$
0=\frac{\partial \Gamma}{\partial \Lambda}=\frac{192 \pi^{2}}{\Lambda^{2}}-\frac{C_{E}}{\Lambda} \quad \Rightarrow \quad \frac{1}{\Lambda_{E}}=\frac{C_{E}}{192 \pi^{2}},
$$

where $\Lambda_{E}$ is the induced cosmological constant one obtains in pure Einsteinian gravity, and $C_{E}$ a gauge-dependent constant which can be easily computed in closed form by using (61), (64). It reads

$$
C_{E}=\frac{\gamma_{0}+\gamma_{1} \gamma+\gamma_{2} \gamma^{2}}{180 \gamma^{2}(3-\rho)^{4}}
$$

$\gamma_{0}=-270\left(259-324 \rho+162 \rho^{2}-36 \rho^{3}+3 \rho^{4}\right.$,

$\gamma_{1}=360\left(297-360 \rho+176 \rho^{2}-36 \rho^{3}+3 \rho^{4}\right.$,

$\gamma_{2}=-388719+537732 \rho-282906 \rho^{2}+66948 \rho^{3}+6479 \rho^{4}$.

A formula for $\Lambda$ analog to the one in (65) is valid also in the presence of the scalar field when $m=0$. In fact, in this case too the $Q_{n}$-functions in (61) do not depend on $\Lambda$, and thus we obtain

$$
\frac{1}{\Lambda}=\frac{C_{E}-\frac{61}{180}}{192 \pi^{2}}=\frac{1}{\Lambda_{E}}-\frac{61}{34560 \pi^{2}}<\frac{1}{\Lambda_{E}} .
$$

From the latter equation we see that the contribution due to the coupling does not depend on the gauge parameters and it increases the cosmological constant, as compared to the one induced by pure Einsteinian gravity.

If $m \neq 0$ the last term in (64) is non-vanishing and the equation for $\Lambda$ is more complicated. We assume the parameter $\alpha$ to be sufficiently small order that the series can be dropped off. Then, we get

$$
\frac{\partial \Gamma}{\partial \Lambda}=\frac{192 \pi^{2}}{\Lambda^{2}}-\frac{C_{E}+\frac{119}{180}}{\Lambda}+\frac{5 m^{2}}{4 \Lambda^{2}}-\frac{m^{2}}{2 \Lambda^{2}} \log \frac{\Lambda}{3 \mu^{2}}+O\left(m^{2} / \Lambda\right),
$$

It needs to be noticed that, in the limit $m \rightarrow 0$, the latter equation does not lead to (68) implying the presence of a zero mode. In fact, when $m=0$, the last term in (54) has a zero-mode which has to be removed in the computation of the corresponding zeta-function. If in (69) we disregard the terms depending on $m$, then the contribution due to the coupling does not depend on the gauge parameters, as in the previous case, but in contrast with that case it decreases the cosmological constant $\Lambda_{E}$.

Let us now turn to the discussion of the second case, Eq. (55), which in general is more complicated than the previous one, due to the presence of the $f$ function, which depends on $\Lambda$. In this case, the one-loop contribution to the effective action can be read off from (55). It has the form

$$
\begin{aligned}
& \Gamma^{(1)}=Q_{0}\left(\frac{9}{4}+\frac{12}{3-\rho}\right)+Q_{1}\left(\frac{25}{4}\right)-\frac{1}{2} Q_{1}\left(\frac{25}{4}-\frac{6\left(f^{\prime} V-1\right)}{\gamma}\right) \\
& -\frac{1}{2} Q_{2}\left(\frac{33}{4}-\frac{12}{f^{\prime} V+1}\right) \\
& -\frac{1}{2} Q_{0}\left(\frac{25}{4}\right)-\frac{1}{2} Q_{0}\left(\frac{9}{4}+q_{1}\right)-\frac{1}{2} Q_{0}\left(\frac{9}{4}+q_{2}\right) .
\end{aligned}
$$

The computation simplifies in the case in which $f^{\prime}$ does not depend on $\Lambda$. This means that one considers only functions linear in the curvature. In such case, none of the arguments of the $Q_{n}$-functions in (70) depends on $\Lambda$ and thus, as in (68), one gets

$$
\frac{\partial \Gamma}{\partial \Lambda}=\frac{192 \pi^{2}}{\Lambda^{2}}-\frac{C}{\Lambda} \quad \Rightarrow \quad \frac{1}{\Lambda}=\frac{C}{192 \pi^{2}} .
$$

where $C=C\left(\gamma, \rho, f^{\prime} V\right)$ is a complicated function which, in principle, can be computed explicitly. Here we write it down only in the Landau gauge.

Starting from (58) we have

$$
\Gamma^{(1)}=\frac{1}{2} Q_{1}\left(\frac{25}{4}\right)-\frac{1}{2} Q_{2}\left(\frac{33}{4}-\frac{12}{f^{\prime} V+1}\right)-\frac{1}{2} Q_{0}\left(\frac{25}{4}\right),
$$


and choosing again $f^{\prime}$ to be a constant we finally get

$$
\frac{1}{\Lambda}=\frac{C}{192 \pi^{2}}, \quad C=\frac{43\left(f^{\prime} V\right)^{2}-114 f^{\prime} V+443}{20\left(f^{\prime} V+1\right)^{2}} .
$$

Thus, we have explicitly found the induced cosmological constant which may be relevant in the inflationary epoch, for several simple variants of non-minimal modified gravity.

\section{INDUCED COSMOLOGICAL CONSTANT IN THE LARGE $R$ LIMIT}

It is interesting to note that quantum corrections may indeed induce a de Sitter universe, which generates the inflationary phase. We then consider the one-loop effective action for a simple, specific model in the large- $R$ limit. This means that we look for de Sitter solutions with very large curvature, which could in fact be induced by quantum corrections. In (49), for simplicity, we set $\beta=0$.

We first consider a simple toy model, quadratic in the curvature, defined by $f(R)=\alpha R^{2}, \alpha$ being a constant. As it follows from (6), such model has only one classical solution with constant curvature, which corresponds to Minkowski one $\left(R_{0}=0\right)$. In the large $R=4 \Lambda$ limit, we trivially get

$$
Z^{(1)}(\gamma, 0, \rho):\left[\operatorname{det}\left(-\Delta_{0}+V^{\prime \prime}\right)\right]^{-1 / 2},
$$

from which it follows that

$$
\begin{gathered}
\Gamma=-384 \pi^{2} \alpha V-\frac{24 \pi^{2}}{\Lambda}-\frac{1}{2} Q_{0}\left(\frac{9}{4}-\frac{3 V^{\prime \prime}}{\Lambda}\right), \\
\frac{\partial \Gamma}{\partial \Lambda}=\frac{192 \pi^{2}}{\Lambda^{2}}+\frac{5 V^{\prime \prime}}{4 \Lambda^{2}}-\frac{V^{\prime \prime}}{2 \Lambda^{2}} \log \frac{\Lambda}{3 \mu^{2}}+O\left(V^{\prime \prime} / \Lambda\right),
\end{gathered}
$$

and this implies that quantum corrections induce a de Sitter solution with large curvature

$$
R=\frac{3}{4 \mu^{2}} \exp \left(\frac{5}{2}+\frac{384 \pi^{2}}{V^{\prime \prime}}\right)
$$

The solution is stable if $V^{\prime \prime}>0$.

As a second example, we choose $f(R)=\alpha R \log \frac{R}{\beta}, \alpha$ and $\beta$ being constants. From (6) it follows that such a model has the following classical de Sitter solutions:

$$
\begin{aligned}
& \text { 1): } R_{0}=0, \quad V_{0} \text { arbitrary, } \\
& \text { 2): } R_{0}=\beta, \quad V_{0}=1 / \alpha \text {, } \\
& \text { 3): } R_{0}=\beta \exp \left(1-\frac{1}{\alpha V_{0}}\right), \quad V_{0}^{\prime}=0 \text {. }
\end{aligned}
$$

The one-loop quantum corrections in the large $R$ limit for this case give

$$
Z^{(1)}(\gamma, 0, \rho):\left[\operatorname{det}\left(-\Delta_{0}+V^{\prime \prime}+\frac{\left[V^{\prime}(\log (R / \beta)-1)\right]^{2}}{V \log (R / \beta)}\right)\right]^{-1 / 2} .
$$

Now, we obtain

One can see (graphically) that there are values of $R=4 \Lambda$ for which the latter expression vanishes. In particular, for very large $R$ the last term is dominant and $\log (4 \Lambda / \beta)$ can be derived in closed form as a function of the potential and its derivatives. In this way we get a de Sitter solution induced by quantum corrections, which is unstable for suitable potentials and could describe the inflationary phase of the universe. Thus, we have demonstrated that quantum effects in non-minimal gravity may induce a large cosmological constant which produces the de Sitter inflation.

$$
\begin{aligned}
\Gamma= & -\frac{24 \pi^{2}}{\Lambda}\left(1+4 \alpha V \log \frac{4 \Lambda}{\beta}\right)-\frac{1}{2} Q_{0}\left(\frac{9}{4}-\frac{3 V^{\prime \prime}}{\Lambda}-\frac{3\left[V^{\prime}(\log (4 \Lambda / \beta)-1)\right]^{2}}{\Lambda V \log (4 \Lambda / \beta)}\right) \\
\frac{\partial \Gamma}{\partial \Lambda}= & -\frac{96 \pi^{2} \alpha V}{\Lambda^{2}}+\frac{24 \pi^{2}}{\Lambda^{2}}\left(1+4 \alpha V \log \frac{4 \Lambda}{\beta}\right) \\
& -\frac{\left[-1.53+\left(\log \frac{4 \Lambda}{\beta}\right)^{2}\left(6.59-3 \log \frac{\Lambda}{3 \mu^{2}}\right)+\log \frac{\Lambda}{3 \mu^{2}}+\log \frac{4 \Lambda}{\beta}\left(-2.53+\log \frac{\Lambda}{3 \mu^{2}}\right)\right]\left(V^{\prime}\right)^{2}}{2 \Lambda^{2} V\left(\log \frac{4 \Lambda}{\beta}\right)^{2}} \\
& -\frac{\left(\log \frac{4 \Lambda}{\beta}\right)^{3}\left(-2.53+\log \frac{\Lambda}{3 \mu^{2}}\right)\left(V^{\prime}\right)^{2}+\left(\log \frac{4 \Lambda}{\beta}\right)^{2}\left(-2.53+\log \frac{\Lambda}{3 \mu^{2}}\right) V V^{\prime \prime}}{2 \Lambda \log \frac{4 \Lambda}{\beta}\left[\left(\log \frac{4 \Lambda}{\beta}-1\right)^{2}\left(V^{\prime}\right)^{2}+V V^{\prime \prime}\right]} \\
& \frac{-1.32\left(\log \frac{4 \Lambda}{\beta}-2.11\right)\left(\log \frac{4 \Lambda}{\beta}-1\right)\left(\log \frac{4 \Lambda}{\beta}+0.36\right)\left(V^{\prime}\right)^{2}+1.32\left(\log \frac{4 \Lambda}{\beta}\right)^{2} V V^{\prime \prime}}{\beta} .
\end{aligned}
$$




\section{CONCLUSION}

In summary, we have discussed in this paper nonminimal modified gravity and its availability for the description of the early- and late-time eras of our universe. The one-loop effective action in the de Sitter background with a constant background scalar field was found. Its gaugefixing independent version has also been obtained. Using the one-loop effective action we derived the effective induced cosmological constant, which may be responsible for inducing the early-time de Sitter epoch. Moreover, it turns out that in some cases when there is no classical de Sitter universe solution, the one-loop quantum effects induce the de Sitter space at the quantum-corrected level. The classical reconstruction scheme for non-minimal modified gravity was developed and it was demonstrated that, by using such a scheme, one can present a convenient model which unifies early-time inflation with late-time acceleration.

It would be interesting to exhibit realistic models of such unification which turns out to be quite complicated in its explicit form. Then, the one-loop calculation may give a way to estimate the precise role of quantum corrections in the early-time inflation. Unfortunately, to realize this program one has to carry out the explicit one-loop calculation on the de Sitter background with a dynamical background scalar, what is not the easy task. In the present work we have been able to do such calculation only in the case when the background scalar is constant.

From another side, the quantum effects of non-minimal modified gravity may be relevant also in the future for the models which develop the classical finite-time singularity. It is to be expected that, in analogy with the results in Refs. $[25,26]$, such quantum gravity effects will drive the future universe to the de Sitter era before the singularity occurs.. In this respect, the one-loop calculation on the de Sitter background can be also relevant for the future dark energy epoch.

\section{ACKNOWLEDGEMENTS}

GC acknowledges the support received from the European Science Foundation (ESF) for the activity entitled "New Trends and Applications of the Casimir Effect" (Exchange Grant 2262) for the period March-April 2009, during which part of the present paper has been written. EE's research was performed in part while on leave at Department of Physics and Astronomy, Dartmouth College, 6127 Wilder Laboratory, Hanover, NH 03755, USA. The work by S.N. is supported by the Global COE Program of Nagoya University provided by the Japan Society for the Promotion of Science (G07). The research by SDO has been supported in part by JSPS (Japan). This paper is partly an outcome of the collaboration program INFN (Italy) and CSIC (Spain). It has been also supported in part by MEC (Spain), project FIS2006-02842 and grant PR2009-0314, by AGAUR (Generalitat de Catalunya), contract 2009SGR-994, and by LRSS, project 2553.2008.2 (Russia).

\section{REFERENCES}

[1] Nojiri S, Odintsov SD. Introduction to modified gravity and gravitational alternative for dark energy. Int J Geom Meth Mod Phys 2007; 4: 115.
[2] Nojiri S, Odintsov SD. Modified gravity with negative and positive powers of the curvature: Unification of the inflation and of the cosmic acceleration. Phys Rev D 2003; 68: 123512.

[3] Cognola G, Elizalde E, Nojiri S, Odintsov SD, Sebastiani L, Zerbini S. Class of viable modified $f(R)$ gravities describing inflation and the onset of accelerated expansion. Phys Rev D 2008; 77 : 046009.

[4] Nojiri S, Odintsov SD, Tretyakov PV. From inflation to dark energy in the non-minimal modified gravity. Prog Theor Phys Suppl 2008; $172: 81$.

[5] Nojiri S, Odintsov SD. Gravity assisted dark energy dominance and cosmic acceleration. Phys Lett B 2004; 599: 137; Nojiri S, Odintsov SD. Dark energy and cosmic speed-Up from consistent modified gravity. PoSWC2004 2004: 024.

[6] Allemandi G, Borowiec A, Francaviglia M, Odintsov SD. Dark energy dominance and cosmic acceleration in first order formalism. Phys Rev D 2005; 72: 063505 .

[7] Mukohyama S, Randall L. A dynamical approach to the cosmological constant. Phys Rev Lett 2004; 92: 211302; Inagaki T, Nojiri $\mathrm{S}$, Odintsov SD. The one-loop effective action in phi**4 theory coupled non-linearly with curvature power and dynamical origin of cosmological constant. J Child Adolesc Psychopharmacol 2005; 0506: 010. Dolgov AD, Kawasaki M. Realistic cosmological model with dynamical cancellation of vacuum energy. arXiv:astro$\mathrm{ph} / 0307442$.

[8] Bertolami O, Boehmer CG, Harko T, Lobo FSN. Extra force in $f(R)$ modified theories of gravity. Phys Rev D 2007; 75: 104016; Bertolami O, Paramos J, Harko T, Lobo FSN. Non-minimal curvaturematter couplings in modified gravity. arXiv:0811.2876 [gr-qc] Koivisto TS. Disformal quintessence. arXiv:0811.1957 [astro-ph]. Puetzfeld D, Obukhov YN. On the motion of test bodies in theories with non-minimal coupling. Phys Rev D 2008; 78: 121501. Harko T. Modified gravity with arbitrary coupling between matter and geometry.' Phys Lett B 2008; 669: 376. Bertolami O, Paramos J. On the non-trivial gravitational coupling to matter. Class Quantum Gravity. 2008); 25: 245017; Nesseris S. Matter density perturbations in modified gravity models with arbitrary coupling between matter and geometry. Phys Rev D 2009; 79: 044015; Borowiec A. from dark energy to dark matter via non-minimal coupling. arXiv:0812.4383 [gr-qc].

[9] Faraoni V. A viability criterion for modified gravity with an extra force. Phys Rev D 2007; 76: 127501. Sotiriou TP, Faraoni V. Modified gravity with R-matter couplings and (non-)geodesic motion. Class Quantum Gravity 2008; 25: 205002; Sotiriou TP. The viability of theories with matter coupled to the Ricci scalar. Phys Lett B 2008; 664: 225 .

[10] Cognola G, Gastaldi M, Zerbini S. On the Stability of a class of Modified Gravitational Models. Int J Theor Phys 2008; 47: 898.

[11] Cognola G, Zerbini S. Homogeneous cosmologies in generalized modified gravity. Int J Theor Phys 2008; 47: 3186.

[12] Nojiri S, Odintsov SD. Modified gravity and its reconstruction from the universe expansion history. J Phys Conf Ser 2007; 66: 012005.

[13] Capozziello S, Nojiri S, Odintsov SD, Troisi A. Cosmological viability of $f(R)$-gravity as an ideal fluid and its compatibility with a matter dominated phase. Phys Lett B 2006; 639: 135. Nojiri S, Odintsov SD. Modified $f(R)$ gravity consistent with realistic cosmology: From matter dominated epoch to dark energy universe. Phys Rev D 2006; 74: 086005.

[14] Nojiri S, Odintsov SD. Unifying phantom inflation with late-time acceleration: scalar phantom-non-phantom transition model and generalized holographic dark energy. Gen Rel Gravity 2006; 38: 1285; Capozziello S, Nojiri S, Odintsov SD. Unified phantom cosmology: inflation, dark energy and dark matter under the same standard. Phys Lett B 2006; 632: 597.

[15] Fujii Y, Maeda K. The scalar-tensor theory of gravitation. Cambridge, USA, 2003; p. 240; Available from: http://www.slac.stanford.edu/ spires/find/hep/www?irn=5550017

[16] Cognola G, Zerbini S. One-loop $f(R)$ gravitational modified models. J Phys A 2006; 39: 6245.

[17] Cognola G, Elizalde E, Nojiri S, Odintsov SD, Zerbini S. One-loop $f(R)$ gravity in de Sitter universe. J Child Adolesc Psychopharmacol 2005; 0502: 10.

[18] Cognola G, Elizalde E, Zerbini S. String-inspired Gauss-Bonnet gravity reconstructed from the universe expansion history and 
yielding the transition from matter dominance to dark energy. Phys Rev D 2007; 75: 086002.

[19] Cognola G, Elizalde E, Nojiri S, Odintsov SD, Zerbini S. One-loop effective action for non-local modified Gauss-Bonnet gravity in de Sitter space. Eur Phys J 2009; 64: 483.

[20] Fradkin ES, Tseytlin AA. One-loop effective potential in gauged $\mathrm{O}(4)$ supergravity and the problem of term. Nucl Phys B 1984; 234: 472 .

[21] Buchbinder IL, Odintsov SD, Shapiro IL. Effective action in quantum gravity. Bristol, UK, 1992. Available from: http://www.slac.stanford.edu/spires/find/hep/www?irn=2762668

[22] Elizalde E, Vanzo L, Zerbini S. Zeta-function regularization, the multiplicative anomaly and the Wodzicki residue. Commun Math Phys 1998; 194: 613.
[23] Bytsenko AA, Cognola G, Vanzo L, Zerbini S. Quantum fields and extended objects in space-times with constant curvature spatial section. Phys Rep 1996; 266: 1.

[24] Elizalde E, Odintsov SD, Romeo A, Bytsenko AA, Zerbini S. Zeta regularization techniques with applications. World Scientific, Singapore, 1994; Elizalde E. Ten Physical applications of spectral zeta functions. Berlin: Springer Verlag 1995.

[25] Abdalla MCB, Nojiri S, Odintsov SD. Consistent modified gravity: dark energy, acceleration and the absence of cosmic doomsday. Class Quantum Gravity 2005; 22: L35.

[26] Elizalde E, Nojiri S, Odintsov SD. Late-time cosmology in (phantom) scalar-tensor theory: dark energy and the cosmic speed-up. Phys Rev D 2004; 70: 043539 .

(C) Cognola et al.; Licensee Bentham Open.

This is an open access article licensed under the terms of the Creative Commons Attribution Non-Commercial License (http://creativecommons.org/licenses/by-nc/3.0/) which permits unrestricted, non-commercial use, distribution and reproduction in any medium, provided the work is properly cited. 